\title{
GRADIENT VECTOR FIELDS WHICH CHARACTERIZE WARPED PRODUCTS
}

\author{
NOBUHIRO INNAMI* and BYUNG HAK KIM**
}

\begin{abstract}
We find what condition on gradient vector fields characterizes warped products, Riemannian products and round spheres. To do this we apply the theory of Jacobi equations without conjugate points to the differential maps of the local one-parameter groups generated by gradient vector fields.
\end{abstract}

\section{Introduction}

Throughout the paper let $M$ be a complete $n$-dimensional Riemannian manifold with Riemannian metric $g$ and without boundary and let $X=\operatorname{grad} f$ be the gradient vector field of a function $f$ on $M$. We assume that $X$ is complete, namely, the domain of any maximal integral curve is by definition the whole real line $(-\infty, \infty)$. The Riemannian curvature tensor $R$ satisfies by definition

$$
\nabla_{X} \nabla X+\nabla X \circ \nabla X+\left(-\nabla \nabla_{X} X+R(\cdot, X) X\right)=0,
$$

where $\nabla$ is the Levi-Civita connection. It holds true that for a gradient vector field $X$

(i) $\nabla X$ is a $(1,1)$-tensor field corresponding to the Hessian of $f$, i.e.,

$$
g\left(\nabla_{Y} X, Z\right)=\operatorname{Hes}_{f}(Y, Z)=Y(Z f)-\left(\nabla_{Y} Z\right) f .
$$

Hence, $\nabla X$ is a symmetric endomorphism of the tangent space $T M$ of $M$.

(ii) $\nabla_{X} X=(1 / 2) \operatorname{grad}\|X\|^{2}$. Hence, $\nabla \nabla_{X} X$ is a symmetric endomorphism of $T M$.

* Partly supported by the Grants-in-Aid for Scientific Research, the Ministry of Education, Science and Culture, Japan

** Partly supported by BSRI-97-1419

Received June 2, 1998 
Therefore, (1) implies that the Hessian endomorphism $\nabla X$ satisfies the endomorphism valued Riccati differential equation

$$
\nabla_{X} U+U^{2}+A=0,
$$

where $A(\cdot)=-\nabla \cdot \nabla_{X} X+R(\cdot, X) X$. The existence of symmetric solutions of (2) defined on any maximal integral curve is sometimes controlled by the property of the symmetric endomorphism $A$. From this point of view we find what condition characterizes warped products. Let $\psi:(a, b) \rightarrow \mathrm{R}$ be a positive function and $M_{0} \times(a, b)$ the product manifold of $M_{0}$ and $(a, b)$. We say that $\left(M_{0} \times(a, b), g\right)$ is a warped product with warping function $\psi$ if the Riemannian metric $g$ on $M_{0} \times(a, b)$ is given by $g_{(q, t)}=\psi(t)^{2} g_{q}+d t^{2}$ for any $(q, t) \in M_{0} \times(a, b)$. The metric of any warped product is represented as $g_{(q, s)}=\bar{\psi}(s)^{2}\left(g_{q}+d s^{2}\right)$ for suitable coordinates. Our studies are based on the following fundamental theorem due to Y. Tashiro. In the following theorem, the number of points with $X=0$ was shown by T. Maebashi ([5]).

Theorem 1 ([8]). Suppose there is a function $h$ on $M$ such that $\nabla X=h I$ where I is the identity endomorphism. Then, the following are true.

(a) There is a function $\ell$ on the image of $f$ such that $h=\ell \circ f$.

(b) The number of points with $X=0$ is zero, one or two. Moreover, $M$ is isometric to a warped product except at points with $X=0$.

(c) If the derivative $\ell^{\prime}$ is a constant function and there is a point $p \in M$ with $X=0$, then $M$ is isometric to a space form which is by definition a simply connected complete manifold of constant curvature.

(d) If $h=0$ on $M$, then $M$ is isometric to a Riemannian product $N \times \mathrm{R}$.

Therefore, our purpose is reduced to a problem of finding what condition on $A$ characterizes the equation $\nabla X=h I$. Let $\operatorname{Ric}(X)$ be the trace of the Riemannian curvature endomorphism $R(\cdot, X) X$. In the light of Sakai's works ([6],[7]) we prove the following Theorems 2 and 3. As contrasted with his works, the different point is that we do not assume $\|X\|=$ const. on $M$.

Theorem 2. Suppose $\operatorname{Ric}(X) \geq \triangle\|X\|^{2} / 2$ for all points $p \in M$ where $\triangle$ is by definition the trace of the Hessian endomorphism. Then, $M$ is isometric to a Riemannian product $N \times \mathrm{R}$, where $N=f^{-1}(0)$.

THEOREM 3. Suppose there is a positive function $z$ along any maximal integral curve of $X$ such that

$$
-\frac{1}{2} \Delta\|X\|^{2}+\operatorname{Ric}(X) \geq-a \frac{z^{\prime \prime}}{z}
$$




$$
\lim _{T \rightarrow \pm \infty} \int_{0}^{T} \frac{1}{z^{2}} d t= \pm \infty
$$

for some constant $a \geq n$. Then, $M$ is isometric to a warped product except for points $p \in M$ with $X=0$ if $a=n$, and $M$ is isometric to a Riemannian product $N \times \mathrm{R}$ if $a>n$.

Corollary 4. Suppose there exist functions $f_{1}, \ldots, f_{n}$ on $M$ such that $X_{i}=\operatorname{grad} f_{i}$ is linearly independent on an open dense subset $V$ in $M$ and they satisfy the assumptions of Theorem 3 under $a=n$. Then $M$ is a space form of non-negative curvature.

Let $p \in M$ and Let $d_{p}: M \rightarrow \mathrm{R}$ be the distance function to the point $p$ on $M$ induced from the Riemannian metric $g$ on $M$, namely, $d_{p}(q)=d(p, q)$ for any $q \in M$. Let $C_{p}$ be the cut locus of $p$ in $M$ (see e.g. [1]) and $V(p)=$ $M-\left(C_{p} \cup\{p\}\right)$. Then, $V(p)$ is open dense set in $M$ and $d_{p}$ is differentiable on $V(p)$.

THEOREM 5. Let $k$ be a constant and let $f: M \rightarrow \mathrm{R}$ be a function given by $f(q)=\cos k d_{p}(q)$ for any $q \in M$. Suppose the gradient vector field $X$ of $f$ in $V(p)$ is complete. If the inequality

$$
-\frac{1}{2} \triangle\|X\|^{2}+\operatorname{Ric}(X) \geq n k^{4}\left(\sin ^{2} k d_{p}-\cos ^{2} k d_{p}\right)
$$

holds true in $V(p)$, then $M$ is isometric to a sphere of radius $1 / k$.

This work began when the first author visited Kyung Hee University in Summer of 1996 and was completed when the second author visited Niigata University in Winter of 1997.

\section{Riccati equations associated with vector fields}

Let $X$ be a complete vector field on $M$ and let $\varphi_{t}: M \rightarrow M$ be the local oneparameter group of diffeomorphisms of $X$. Let $p \in M$ and $c_{p}:(-\infty, \infty) \rightarrow$ $M$ the maximal integral curve of $X$ with $c_{p}(0)=p$. Let $e_{1}(t), \ldots, e_{n}(t)$ be a parallel orthonormal basis of $T_{c_{p}(t)} M$ along $c_{p}$. The tangent vectors, $\nabla X$, $R(\cdot, X) X$, etc. will be represented with respect to the basis $e_{1}(t), \ldots, e_{n}(t)$ by $n$-tuples, $n \times n$-matrices, etc. Let $D_{p}(t)$ be the matrix representation of $d \varphi_{t} \circ P_{t}^{-1}$ for any $t \in(-\infty, \infty)$, where $d \varphi_{t}$ is the differential map of $\varphi_{t}$ from $T_{p} M$ to $T_{c_{p}(t)} M$ and $P_{t}$ is the parallel translation along $c_{p}$ from $T_{p} M$ to $T_{c_{p}(t)} M$. If $U(t)$ is the matrix representation of $\nabla X$ at $c_{p}(t)$, then

$$
D_{p}^{\prime}(t)=U(t) D_{p}(t)
$$


for all $t \in(-\infty, \infty)$. The equation (1) in Section 1 is written as

$$
U^{\prime}(t)+U(t)^{2}+A(t)=0
$$

where $A(t)$ is the matrix representation of $-\nabla \nabla_{X} X+R(\cdot, X) X$ at $c_{p}(t)$, and, $D_{p}(t)$ satisfies the Jacobi equation

$$
D_{p}^{\prime \prime}(t)+A(t) D_{p}(t)=0 .
$$

Notice that if $X$ is the gradient vector field of a function $f$, then $U(t)$ is a symmetric matrix for every $t \in(-\infty, \infty)$. Hence, $\left(\mathrm{J}_{\mathrm{A}}\right)$ is disconjugate on $(-\infty, \infty)$ (see Fact 1 in Section 3).

\section{Solutions of Riccati equations}

Let $L(n)$ and $S(n)$ be the set of all $n \times n$-matrices and symmetric ones, respectively. Let $A:(-\infty, \infty) \rightarrow S(n)$ be a differentiable map. Consider the $L(n)$-valued Riccati equation

$\left(\mathrm{R}_{\mathrm{A}}\right)$

$$
U^{\prime}+U^{2}+A=0
$$

and the $L(n)$-valued Jacobi equation

$$
D^{\prime \prime}+A D=0 .
$$

We say that $\left(\mathrm{J}_{\mathrm{A}}\right)$ is disconjugate on $(-\infty, \infty)$ if any solution of $\left(\mathrm{J}_{\mathrm{A}}\right)$ with $D\left(t_{0}\right)=0$ and det $D^{\prime}\left(t_{0}\right) \neq 0$ for given $t_{0}$ satisfies that det $D(t) \neq 0$ for any $t \neq t_{0}$. In the below $\left(\mathrm{J}_{\mathrm{k}}\right)$ and $\left(\mathrm{R}_{\mathrm{k}}\right)$ mean the real valued differential equations of the same types as $\left(\mathrm{J}_{\mathrm{A}}\right)$ and $\left(\mathrm{R}_{\mathrm{A}}\right)$ in which $A$ is replaced by a function $k$. In the present note we use the following facts.

FACT 1. The equation $\left(\mathrm{J}_{\mathrm{A}}\right)$ is disconjugate on $(-\infty, \infty)$ if and only if there is a symmetric solution of $\left(\mathrm{R}_{\mathrm{A}}\right)$ defined on $(-\infty, \infty)$.

For the proof see Theorem 10.2 of Chpter XI in [3].

FACT 2. Let $k:(-\infty, \infty) \rightarrow \mathrm{R}$ be a function such that $\left(\mathrm{J}_{\mathrm{k}}\right)$ is disconjugate on $(-\infty, \infty)$ and $\operatorname{tr} A(t) \geq n k(t)$ for all $t \in(-\infty, \infty)$. Suppose $\left(\mathrm{J}_{\mathrm{A}}\right)$ is disconjugate on $(-\infty, \infty)$. Then, we have that

$$
n u(t) \leq \operatorname{tr} U(t) \leq \operatorname{tr} U_{1}(t) \leq \operatorname{tr} \tilde{U}(t) \leq n \tilde{u}(t),
$$

where $u(t)$ and $\tilde{u}(t)$ are the minimal and maximal solutions of $\left(\mathrm{R}_{\mathrm{k}}\right)$ defined on $(-\infty, \infty)$, respectively, $U(t)$ and $\tilde{U}(t)$ are the minimal and maximal symmetric solutions of $\left(\mathrm{R}_{\mathrm{A}}\right)$ defined on $(-\infty, \infty)$, respectively, $U_{1}(t)$ is any symmetric 
solution of $\left(\mathrm{R}_{\mathrm{A}}\right)$ defined on $(-\infty, \infty)$. The equality sign holds true in the first inequality or fourth one for all $t \in(-\infty, \infty)$ if and only if $U(t)=u(t) I$ or $\tilde{U}(t)=\tilde{u}(t) I$, and $A(t)=k(t) I$ for all $t \in(-\infty, \infty)$, where $I$ is the identity matrix. In particular, every equality sign in the above holds true if $\left(\mathrm{R}_{\mathrm{k}}\right)$ has a unique solution defined on $(-\infty, \infty)$. In this case, $\left(\mathrm{R}_{\mathrm{A}}\right)$ has also a unique symmetric solution defined on $(-\infty, \infty)$.

SKETCH OF THE PROOF. Let $D_{s}(t)$ (and $f_{s}(t)$ ) be solutions of $\left(\mathrm{J}_{\mathrm{A}}\right)$ (and $\left.\left(\mathrm{J}_{\mathrm{k}}\right)\right)$ with $D_{s}(s)=0, D_{s}{ }^{\prime}(s)=I$ (and $f_{s}(s)=0, f_{s}{ }^{\prime}(s)=1$, resp. ) on $(-\infty, \infty)$ and let $U_{s}(t)=D_{s}{ }^{\prime}(t) D_{s}(t)^{-1}$ and $u_{s}(t)=f_{s}{ }^{\prime}(t) f_{s}(t)^{-1}$ for all $t \neq s$. We can prove in the same way as the Rauch comparison theorem (see e.g. p.32 of [1]) that $\operatorname{tr} U_{s}(t) \geq n u_{s}(t)$ for all $t>s$ and $\operatorname{tr} U_{s}(t) \leq n u_{s}(t)$ for all $t<s$. By construction $U_{s}(t)$ and $u_{s}(t)$ are maximal (and minimal) for $t<s$ (and $t>s$, resp.) in the set of all symmetric solutions of $\left(\mathrm{R}_{\mathrm{A}}\right)$ and $\left(\mathrm{R}_{\mathrm{k}}\right)$ defined on those domains. The limit solutions as $s \longrightarrow \pm \infty$ satisfy the inequality in Fact 2.

FACT 3. Let a be a constant with $a \geq n$ and let $k(t)=\operatorname{tr} A(t) / a$ for any $t \in(-\infty, \infty)$. If $\left(\mathrm{J}_{\mathrm{A}}\right)$ is disconjugate on $(-\infty, \infty)$, then $\left(\mathrm{J}_{\mathrm{k}}\right)$ is disconjugate on $(-\infty, \infty)$.

Proof. Let $U(t)$ be a symmetric solution of $\left(\mathrm{R}_{\mathrm{A}}\right)$ on $(-\infty, \infty)$ and $h(t)=\operatorname{tr} U(t) / a$ for any $t \in(-\infty, \infty)$. Set $z(t)=\exp \int_{0}^{t} h(s) d s$ for any $t \in(-\infty, \infty)$. Differentiating $z$ twice, we have the equation

$$
z^{\prime \prime}(t)+\left(k(t)+\frac{\operatorname{tr} U(t)^{2}}{a}-\left(\frac{\operatorname{tr} U(t)}{a}\right)^{2}\right) z(t)=0
$$

for all $t \in(-\infty, \infty)$. Since the differential equation (5) has a positive solution $z(t)$, it is disconjugate on $(-\infty, \infty)$. We see that $\left(\mathrm{J}_{\mathrm{k}}\right)$ is disconjugate on $(-\infty, \infty)$, since

$$
\frac{\operatorname{tr} U(t)^{2}}{a}-\left(\frac{\operatorname{tr} U(t)}{a}\right)^{2} \geq 0
$$

for all $t \in(-\infty, \infty)$ and the differential equation $\left(\mathrm{R}_{\overline{\mathrm{k}}}\right), \bar{k}(t)=k(t)+$ $\operatorname{tr} U(t)^{2} / a-(\operatorname{tr} U(t) / a)^{2}$, has a solution $\bar{u}=z^{\prime} / z$ defined on $(-\infty, \infty)$ (see e.g. Theorem 7.2 of Chapter XI in [3]). This implies Fact 3.

FACT 4. Let $k:(-\infty, \infty) \rightarrow \mathrm{R}$ be a function such that $\left(\mathrm{J}_{\mathrm{k}}\right)$ is disconjugate on $(-\infty, \infty)$. Then, $\left(\mathrm{J}_{\mathrm{k}}\right)$ has a positive solution $z(t)$ on $(-\infty, \infty)$ such that

$$
\lim _{T \rightarrow \pm \infty} \int_{0}^{T} \frac{1}{z(t)^{2}} d t= \pm \infty
$$


if and only if $\left(\mathrm{R}_{\mathrm{k}}\right)$ has a unique solution defined on $(-\infty, \infty)$. Therefore, $\left(\mathrm{R}_{\mathrm{A}}\right)$ has a unique symmetric solution defined on $(-\infty, \infty)$ if $\left(\mathrm{J}_{\mathrm{A}}\right)$ is disconjugate on $(-\infty, \infty)$ and $\operatorname{tr} A(t) \geq n k(t)$ for any $t \in(-\infty, \infty)$.

Proof. Theorem 6.4 of Chapter XI in [3] states that $\left(\mathrm{J}_{\mathrm{k}}\right)$ has a unique positive solution defined on $(-\infty, \infty)$ up to a constant factor. This proves the first part and the second part is due to Fact 2.

We will see that $M$ is isometric to a warped product except for points $p$ in $M$ with $X=0$ if $\left(\mathrm{R}_{\mathrm{k}}\right), k=\operatorname{tr} A / n$, has a unique solution defined on $(-\infty, \infty)$ by use of Theorem 3, Facts 3 and 4. Moreover, $M$ is isometric to a Riemannian product $N \times \mathrm{R}$ if $\left(\mathrm{R}_{\mathrm{k}}\right), k=\operatorname{tr} A / a(a>n)$, has a unique solution defined on $(-\infty, \infty)$.

It should be noted that for a negative constant $k=-a^{2}, a>0$, there exists no positive solution $z(t)$ of $\left(\mathrm{J}_{\mathrm{k}}\right)$ satisfying the integral condition in Fact 4 , since the genaral solution of $\left(\mathrm{J}_{\mathrm{k}}\right)$ is $z(t)=C_{1} e^{a t}+C_{2} e^{-a t}$ where $C_{1}$ and $C_{2}$ are constants. Therefore we cannot have hyperbolic analogues of Corollary 4 and Theorem 5 (see Lemma 7).

\section{Proofs of Theorems 2,3 and 5}

As was seen in Section 3, the Jacobi equation associated with a complete gradient vector field $X$ is disconjugate along every maximal integral curve $c_{p}$ of $X$.

Proof of Theorem 2. Fact 4 and Fact 2 prove that $\nabla X=0$ on $M$ since $\left(\mathrm{R}_{0}\right)$ has a unique solution $u(t)=0$ for all $t \in(-\infty, \infty)$. From this we have Theorem 2 (see (d) in Theorem 1, [4], [7], [8]).

Proof of Theorem 3. Case $a=n$ : Facts 2 and 4 imply that $\nabla X=h I$ where $h\left(c_{p}(t)\right)=z^{\prime}(t) / z(t)$ which is independent of the choice of $c_{p}$, and, hence $h$ is a function on $M$. Therefore, Theorem 3 follows from Theorem 1 .

Case $a>n$ : Let $k(t)=\operatorname{tr} A / a, \operatorname{tr} A=-\Delta\|X\|^{2} / 2+\operatorname{Ric}(X)$, and $h(t)=$ $\operatorname{tr} A / a+\operatorname{tr}(\nabla X \circ \nabla X) / a-(\operatorname{tr} \nabla X / a)^{2}$ along any maximal integral curve $c_{p}$. As was seen in the proof of Fact 3 , we see that $\left(\mathrm{J}_{\mathrm{h}}\right)$ is disconjugate on $(-\infty, \infty)$. Facts 2,3 and 4 state that $\left(\mathrm{R}_{\mathrm{k}}\right)$ has a unique solution defined on $(-\infty, \infty)$. Since $k(t) \leq h(t)$ for all $t \in(-\infty, \infty)$, Fact 2 implies that $k(t)=h(t)$ for any $t \in(-\infty, \infty)$, and, hence, we get $\nabla X=0$. Theorem 1 proves that $M$ is isometric to a Riemannian product $N \times \mathrm{R}$.

Proof of Theorem 5. Let $c:(-\infty, \infty) \rightarrow M$ be the maximal integral curve of $X$ with $c(0)=p$. Then, $\dot{c}(t)=-\left(k \sin k d_{p}(c(t))\right) \operatorname{grad} d_{p}(c(t))$ for all $t \in(-\infty, \infty)$. Set $u(t)=-k^{2} \cos k d_{p}(c(t))$ for all $t \in(-\infty, \infty)$. 
Differentiating $u$ at $t$, we get

$$
u^{\prime}(t)=-k^{4} \sin ^{2} k d_{p}(c(t))
$$

for all $t \in(-\infty, \infty)$, since

$$
d_{p}(c(t))^{\prime}=g\left(\dot{c}(t), \operatorname{grad} d_{p}\right)=-k \sin k d_{p}(c(t)) .
$$

Therefore, we have

$$
u^{\prime}(t)+u(t)^{2}+k^{4}\left(\sin ^{2} k d_{p}(c(t))-\cos ^{2} k d_{p}(c(t))\right)=0
$$

for all $t \in(-\infty, \infty)$. Let $F(t)=k^{4}\left(\sin ^{2} k d_{p}(c(t))-\cos ^{2} k d_{p}(c(t))\right)$ for any $t \in(-\infty, \infty)$. We have only to find a solution $z(t)$ of $\left(\mathrm{J}_{\mathrm{F}}\right)$ satisfying the assumption (4) of Theorem 3 , because it proves that $\ell(t)=-k^{2} t$ under the notation in Theorem 1 . Set $z(t)=\exp \int_{0}^{t} u(t) d t$ for any $t \in(-\infty, \infty)$. Then, $z$ is a positive solution of $\left(\mathrm{J}_{\mathrm{F}}\right)$, and, furthermore, we see that

$$
z(t)=\exp \int_{0}^{t}-k^{2} \cos k d_{p}(c(t)) d t=\frac{\sin k d_{p}(c(t))}{\sin k d_{p}(c(0))}
$$

for all $t \in(-\infty, \infty)$, and, hence, $z$ satisfies (4) in Theorem 3. Facts 2, 4 and Theorem 1 prove Theorem 5.

\section{Proofs of Theorem 1 and Corollary 4}

We first prove the following lemma.

LemMA 6. Let $X$ be a complete gradient vector field with $\nabla X=h I$ and let $c_{p}:(-\infty, \infty) \rightarrow M$ be the maximal integral curve of $X$ with $c_{p}(0)=p$. Then, the following hold true.

(a) Any integral curve of $X$ is a reparametrization of a geodesic in $M$.

(b) The length $\|X\|$ is constant in each connected component of any level hypersurface of $f$.

(c) If $p \in M$ and $q \in M$ are such that they are in the same connected component of the level hypersurface $a=f(p)=f(q)$ of $f$, then $f\left(c_{p}(t)\right)=f\left(c_{q}(t)\right)$ for any $t \in(-\infty, \infty)$.

(d) $R(\cdot, X) X=g(\operatorname{grad} h, \cdot) X-g(\operatorname{grad} h, X) I$.

(e) The function $h$ is constant in each level hypersurface of $f$ with $\|X\| \neq 0$. Therefore, there is a function $\ell$ such that $h=\ell \circ f$.

Proof. (a): Since $\nabla_{X} X=h X$, (a) is clear because of the definition of geodesics. 
(b): This is because

$$
Y g(X, X)=2 g\left(\nabla_{Y} X, X\right)=2 h g(Y, X)=0
$$

for any tangent vector $Y$ to the level hypersurface of $f$ which is perpendicular to $X$.

(c): Let $b(s)$ be a curve in the level hypersurface of $f$ such that $b(0)=p$ and $b(1)=q$ and let $\varphi(t, s)$ be the variation of $c_{p}$ through integral curves of $X$ such that $\varphi(t, s)=c_{b(s)}(t)$. Let $Y(t, s)=\frac{\partial \varphi}{\partial s}(t, s)$ and $X(t, s)=\frac{\partial \varphi}{\partial t}(t, s)$. We have only to prove that $g(Y, X)=0$, since

$$
\frac{\partial f \circ \varphi}{\partial s}(t, s)=g(X, Y)
$$

and

$$
f\left(c_{q}(t)\right)-f\left(c_{p}(t)\right)=\int_{0}^{1} \frac{\partial f \circ \varphi}{\partial s}(t, s) d s .
$$

Set $a(t, s)=g(Y(t, s), X(t, s))$. By construction of $b$ we have that $a(0, s)=$ 0 . Since

$$
\frac{\partial a}{\partial t}=g\left(\nabla_{X} Y, X\right)+g\left(Y, \nabla_{X} X\right)=2 h g(Y, X)=2 h a,
$$

it follows from the uniqueness of the solution that $a(t, s)=0$.

(d): The direct computation by using the equation of the Riemannian curvature tensor proves $(\mathrm{d})$.

(e): By (d) we see that

$$
0=g(R(Y, X) X, X)=g(\operatorname{grad} h, Y)\|X\|^{2}
$$

for any tangent vector $Y$ perpendicular to $X$. Therefore, $h$ is constant in the connected component of the level hypersurface of $f$ with $\|X\| \neq 0$.

Proof of Theorem 1. Let $N$ be the set of all points $q \in M$ with $X(q) \neq 0$. Let $p \in N$ and $a=f(p)$. By (b) in Lemma 6 , the connected component $N_{a}$ of the $a$-level hypersurface of $f$ containing $p$ is contained in $N$. Let $q \in N_{a}$ and $c_{q}:(-\infty, \infty) \rightarrow M$ be the maximal integral curve of $X$. Then, from $\left(\mathrm{L}_{\mathrm{U}}\right)$ and $(\mathrm{e})$ in Lemma 6 we get the equation

$$
D^{\prime}(q, t) D(q, t)^{-1}=\nabla X=h\left(c_{q}(t)\right) I=\ell\left(f\left(c_{q}(t)\right)\right) I .
$$

Notice that $h(t):=\ell\left(f\left(c_{q}(t)\right)\right)$ depends only on the point $c_{q}(t)$, and is independent of $q \in N_{a}$. Thus, we have that

$$
D(q, t)=\left(\exp \int_{0}^{t} h(t) d t\right) I
$$


for any point $q \in N_{a}$ and $t \in(-\infty, \infty)$. Let $v, w \in T_{\varphi(q, t)} M$ and let $v_{0}, w_{0} \in$ $T_{q} M$ such that $v=d \varphi_{t}\left(v_{0}\right), w=d \varphi_{t}\left(w_{0}\right)$. Let $v_{0}(t)$, and $w_{0}(t)$ be parallel vector fields along $c_{q}$ with $v_{0}(0)=v_{0}, w_{0}(0)=w_{0}$. Then,

$$
\begin{aligned}
g\left(c_{q}(t)\right)(v, w) & =g\left(c_{q}(t)\right)\left(D(q, t) v_{0}(t), D(q, t) w_{0}(t)\right) \\
& =\left(\exp \int_{0}^{t} h(t) d t\right)^{2} g(q)\left(v_{0}, w_{0}\right) \\
& =\left(\exp \int_{0}^{t} h(t) d t\right)^{2}\left(g_{a}+k^{2} d t^{2}\right)\left(v_{0}, w_{0}\right),
\end{aligned}
$$

where $g_{a}$ is the induced Riemannian metric on the $a$-level hypersurface from $g$ and $k^{2}=\|X\|^{2}>0$ on $N_{a}$. This implies that $M$ is isometric to a Riemannian product $N_{a} \times \mathrm{R}$ if $h=0$ on $M$, and, hence (d) is proved. If $M-N=\emptyset$, then there is no point with $X=0$. Assume $M-N \neq \emptyset$. Since $X\left(c_{q}(t)\right)=$ $D(q, t) X(q)$, we have that $D(q,-\infty)=0$ or $D(q, \infty)=0$ for some $q \in N_{a}$, and, hence, for all $q \in N_{a}$. This implies that the number of points with $X=0$ is one or two. These complete the proof of (b) in Theorem 1. Combining this and (e) in Lemma 6, we get (a) in Theorem 1. Assume that the derivative $\ell^{\prime}$ is constant, say $-k$. By (d) in Lemma6, we have

$$
R\left(\cdot, \frac{X}{\|X\|}\right) \frac{X}{\|X\|}=k I
$$

on the subspace $X^{\perp}$ perpendicular to $X$. Combining this and (a) in Lemma 6, we conclude (c) in Theorem 1 , since there is a point $p \in M$ with $X=0$ which becomes the center of polar coordinates.

The following lemma is needed to prove Corollary 4 and shows that hyperbolic analogue of Theorem 5 is not automatically given.

Lemma 7. Assume $M$ is a space form of negative curvature $k=-a^{2}$. Then there is no nontrivial function $f$ on $M$ such that $D_{q}(t)=z(t) I$ and

$$
\lim _{T \rightarrow \pm \infty} \int_{0}^{T} \frac{1}{z(t)^{2}} d t= \pm \infty
$$

for all $q \in M$ with $X(q) \neq 0$.

Proof. Suppose there exists a nontrivial function $f$ on $M$ such that $D_{q}(t)=z(t) I$ for all $q \in M$ with $X(q) \neq 0$. Then, $\nabla X=\left(z^{\prime}(t) / z(t)\right) I=$ : $h I$. By (d) and (e) in Lemma 6 we have $\ell^{\prime}(t)=a^{2}$, and, therefore, $\nabla X=a^{2} f I$. If $s=\int_{0}^{t}\left\|X\left(c_{q}(t)\right)\right\| d t$ and $\gamma_{q}(s)=c_{q}(t)$ for all $t \in(-\infty, \infty)$, then $\gamma_{q}$ is a 
unit speed geodesic in $M$ (see (a) in Lemma 6). This shows that

$$
D_{q}(t)=z(t) I=\left(C_{1} e^{a s}+C_{2} e^{-a s}\right) I
$$

for all $t \in(-\infty, \infty)$ where $C_{1}+C_{2}=1, C_{1} \geq 0$, and $C_{2} \geq 0$. Since $X\left(c_{q}(t)\right)=z(t) X(q)$, we have $z(t)=\left\|X\left(c_{q}(t)\right)\right\| /\|X(q)\|$ for all $t \in$ $(\infty, \infty)$. Hence

$$
\int_{0}^{t} \frac{1}{z(t)^{2}} d t=\frac{1}{\|X(q)\|} \int_{0}^{s} \frac{1}{z(s)^{3}} d s
$$

for all $t \in(-\infty, \infty)$. Since at least one of $C_{i}$ 's is positive and $z(s) \geq$ $\max \left\{C_{1} e^{a s}, C_{2} e^{-a s}\right\}$ for all $s \in(-\infty, \infty)$, it is impossible that $\lim _{s \rightarrow \pm \infty} \int_{0}^{s}\left(1 / z(s)^{3}\right) d s= \pm \infty$. This completes the proof of Lemma 7.

Proof of Corollary 4. If $n=2$, Theorem 3 proves that $M$ is a warped product except at points with $X_{i}=0$ for each $i=1,2$, and, hence, there exist two one-parameter groups of isometries on $M$. Therefore, $M$ is locally a homogeneous space, and, in particular, the Gauss curvature of $M$ is nonnegative constant because of $\operatorname{dim} M=2$ and Lemma 7.

Suppose $n \geq 3$. By Schur's lemma (see e.g. [2]), we have only to prove that

$$
R(\cdot, v) v=k(p) I
$$

on the subspace $v^{\perp}$ perpendicular to any unit tangent vector $v \in T_{p} M$ and any point $p \in V$. Since $X_{1}(p), \ldots, X_{n}(p)$ is a basis of $T_{p} M$, there exist $a_{1}, \ldots, a_{n}$ such that $v=\sum_{i=1}^{n} a_{i} X_{i}(p)$. From the proof of Theorem 3, there is a function $h_{i}$ on $M$ such that $\nabla X_{i}=h_{i} I$ for each $i=1, \ldots, n$. Let $f: M \rightarrow \mathrm{R}$ be a function given by

$$
f(q)=\sum_{i=1}^{n} a_{i} f_{i}(q)
$$

for any $q \in M$ and $X=\operatorname{grad} f$. Then, we have

$$
X(p)=\sum_{i=1}^{n} a_{i} X_{i}(p)=v
$$

and

$$
\nabla X(q)=\left(\sum_{i=1}^{n} a_{i} h_{i}(q)\right) I .
$$

Combining this equation with (d) and (e) of Lemma 6, we have

$$
R(\cdot, v) v=h_{v} I
$$


on $v^{\perp}$ for some constant $h_{v}$. It remains to prove that $h_{v}$ is independent of the choice of unit vectors $v$ in $T_{p} M$, namely, $h_{v}=h_{w}$ for any unit vectors $v, w \in T_{p} M$. Since $n \geq 3$, we can take a vector $z \in T_{p} M$ with $v \perp z$ and $w \perp z$. By using the vector $z$, we have

$$
\begin{aligned}
h_{v} & =g(R(z, v) v, z)=g(R(v, z) z, v)=h_{z} \\
& =g(R(w, z) z, w)=g(R(z, w) w, z)=h_{w}
\end{aligned}
$$

Theorem 1 implies that $M$ is simply connected if $M$ has constant sectional curvature. If $M$ has negative curvature, then the assumption (4) in Theorem 3 is not satisfied because of Lemma 7. This completes the proof of Corollary 4.

\section{REFERENCES}

1. J. Cheeger and D. Ebin, Comparison Theorems in Riemannian Geometry, North-Holland, Amsterdam, 1975.

2. B.-y. Chen, Geometry of Submanifolds, Marcel Dekker, New York, 1973.

3. P. Hartman, Ordinary Differential Equations, John Wiley \& Sons, 1973.

4. N. Innami, The n-plane with integral curvature zero and without conjugate points, Proc. Japan Acad. Ser. A Math. Sci. 62 (1986), 282-284.

5. T. Maebashi, Vector fields and space forms, J. Fac. Sci. Hokkaido Univ. 15 (1960), 62-92.

6. T. Sakai, On Riemannian manifolds admitting a function whose gradient is of constant norm, Kodai Math. J. 19,1 (1996), 39-51.

7. T. Sakai, Warped products and Riemannian manifolds admitting a function whose gradient is of constant norm, Preprint.

8. Y. Tashiro, Complete Riemannian manifolds and some vector fields, Trans. Amer. Math. Soc. 117 (1965), 251-275.

9. F. Warner, Foundations of Differentiable Manifolds and Lie Groups, Scott, Foresman and Company, Illinois, 1971

DEPARTMENT OF MATHEMATICS AND INFORMATION GRADUATE SCHOOL OF SCIENCE AND TECHNOLOGY NIIGATA UNIVERSITY

NIIGATA, 950-2181

JAPAN

E-mail: innami@math.sc.niigata-u.ac.jp
DEPARTMENT OF MATHEMATICS AND INSTITUTE OF NATURAL SCIENCES

KYUNG HEE UNIVERSITY

SUWON 449-701

KOREA

E-mail: bhkim@nms.kyunghee.ac.kr 